\title{
Event-based control for Sit-to-Stand transition using a Wearable Exoskeleton*
}

\author{
Vijaykumar Rajasekaran, Manuel Vinagre and Joan Aranda, Member, IEEE
}

\begin{abstract}
Sit-to-stand transition is an essential step in a lower limb rehabilitation therapy, mainly for assisting the patient to transit from wheel chair to the next level of therapy. A mixed stiffness-damping control adaptation is proposed for this task which will help in reaching the final position with a constant velocity. A combination of control model is proposed to ensure the initiation and the final stage of the transition, such as to ensure stability and to maintain the equilibrium. The combined control model helps in reaching the goal position with equal participation from the user. For patient studies, such as with paraplegic patients, a combinational control model with muscle stimulation can be included to provide a complete assistance. The role of muscle stimulation and joint movement assistance is also considered in this control model. Further, final stage of this transition must ensure keeping or helping the user to maintain the upright position.
\end{abstract}

Keywords-exoskeleton; wearable robots; sit-to-stand transition; stiffness-damping control;

\section{INTRODUCTION}

Rehabilitation for lower extremity is one of the widely researched topics mainly because of its importance in assisting the user to be independent in pursuing their activities for daily living. In rehabilitation robotics research scenario, the challenges are providing the suitable assistance and to handle the inevitable task of maintaining the equilibrium. Most rehabilitation robotic devices mainly focus on providing the gait assistance which is related to maintaining the center of mass (COM) while performing a dynamic task. However, there are some prerequisite movements or capabilities which are needed before progressing towards the gait, such as balance stability [1], sit-tostand [2].

Sit-to-stand is one of the basic movement and essential part in activities for daily life. This movement also needs the high performance from the user, in lifting

\footnotetext{
*This research is supported by the project AURORA, DPI201570415-C2-1-R, Distributed control strategies and human-robot cooperation in health care settings, funded by the Spanish Ministry of Economy and Competivity, MINECO.

V. Rajasekaran is with the School of Computer science in the University of Birmingham, UK. (Email: v.rajasekaran@bham.ac.uk). M. Vinagre and J. Aranda are associated with the Department of Automatic and Control in the Universitat Politècnica de Catalunya, Barcelona-Tech, Spain. (Email: j.aranda@upc.edu).
}

up the entire upper body weight and simultaneously maintaining the equilibrium of the body. The muscle power in the knee joint plays a key role in this movement and it is essential to provide adequate force assistance for the knee joint [3]. In some cases, an external support is also needed to help the patient maintain the equilibrium and to make a smooth transition [4]. The external support can be provided by means of supporting the upper limb or providing muscle stimulation. In case of paraplegic patients, the role of a coupled system with muscle stimulation and external support in the sit-stand transition has been found efficient in providing the suitable assistance [5]. Wearable sensors based sit-to-stand and stand-to-sit movement was established using the surface EMG signals [6]. The EMG signals are used to monitor the muscle synergies in both the movement and then a fuzzy control model was established to assist the movement. The use of joint torque/force performance instead of independent muscle information or using wearable force/torque sensors has proven to be influential in this specific task [2]. A Smart mobile walker based assistance for elderly people has been performed [7, 8] based on the kinematic computations. A compliant actuator exoskeleton MIRAD [9] was developed specifically for performing the Sit-to-stance movement with a total of 6 flexion and extension movements. A COM based sit-to-stand model is also efficient in ensuring stability above the foot while sitting [10], but a complete kinematic model is needed to ensure its performance. Although, the sit-to-stand is a transition phase, it needs to be handled more precisely to ensure a stable posture at the end.

In this work, a stiffness-damping coupled control model is presented and evaluated for the sit-to-stand task. The control model adaptation is performed in real time with specific consideration to the different stages/events in this rehabilitation task.

\section{EVENT-BASED CONTROL}

Even though, the transition from a sit to stand is considered to be less trivial in most of the rehabilitation robotic studies, it is complex enough to be planned as a single procedure. Although, the initial and final positions in this task are well established it cannot be treated from a position trajectory generation perspective. This solution would imply to a forced movement towards the patient. The user needs to control the start of the action, triggering it by own 


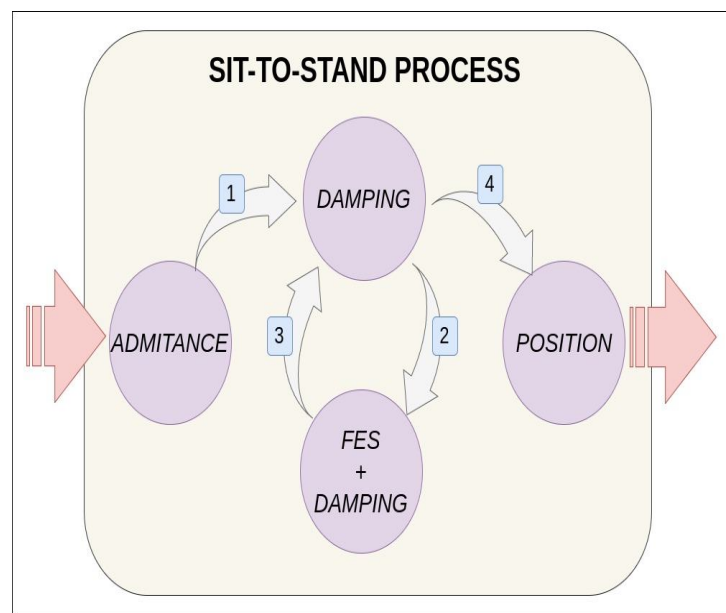

Figure 1. Schematic representation of the switching control model in the sit-to-stand process

volition, since the whole movement requires balance control, involving the upper body dynamics.

In this work, an assist-as-needed strategy which dynamically varies the damping and stiffness variables is adapted. The system must help the exoskeleton users to stand-up, without any prior knowledge about their weight or height. Further, the ability to tackle with parallel activation of functional electrical stimulation (FES) is considered.

Three different stages with associated control strategies have been linked to provide the desired behavior of the system, as shown in Fig.1.

Stage 1: User initiation- An admittance control strategy is considered in this stage, which facilitates the user initiation by giving some freedom in the movement. The joint movement will be initiated when the patient tries to move from the idle or sitting stage to a different position, indicating the intention of the movement. When the controller observes the change in the joint positions and simultaneously observe a change in the neuromotor behavior, initializes the trigger to stage two.

Stage 2: Transition-A damping control strategy is applied based on the error in velocity from which the required resistive or assistive torques is generated, leading to achieve the equilibrium position.

The proposed velocity-based damping controller is represented by the following equation,

$$
\tau=K_{d}\left(\dot{\theta}_{\text {ref }}-\dot{\theta}_{a c t}\right)
$$

where, $\tau$ is the applied torque to assist or resist the joint, $K_{d}$ is the damping constant for the torque acting on the joint. $\dot{\theta}_{r e f}$ and $\dot{\theta}_{a c t}$ are the reference and current joint velocity respectively. In this case, the damping $K_{d}$ is maintained constant to ensure equilibrium and the velocity varies depending on the user movement.
The reference velocity will be specifically programmed for every patient. It will follow a trapezoidal profile generating a smooth transit curve (take-off, constant speed and arrival phases) that helps the transition between the different kinds of control. The trapezoidal profile is asymmetrical demanding lesser acceleration during take-off period in order to gain speed against gravity. When the movement achieves the constant speed reference, FES can be activated applying additional torque to the joint. The effect of FES stimulation acting on a muscle is considered as an impulsive signal [11, 12, 13] (Fig.2(c)). The FES signal must be treated as a disturbance from the control point of view, but in fact, FES action reduces the error in velocity and relaxes the assistance provided by the joint motor, as shown in Fig.2(a). This motor assistance is always positive because the reference velocity is programmed to be higher than that induced by FES action. After reaching the arrival phase, FES must be deactivated to permit the

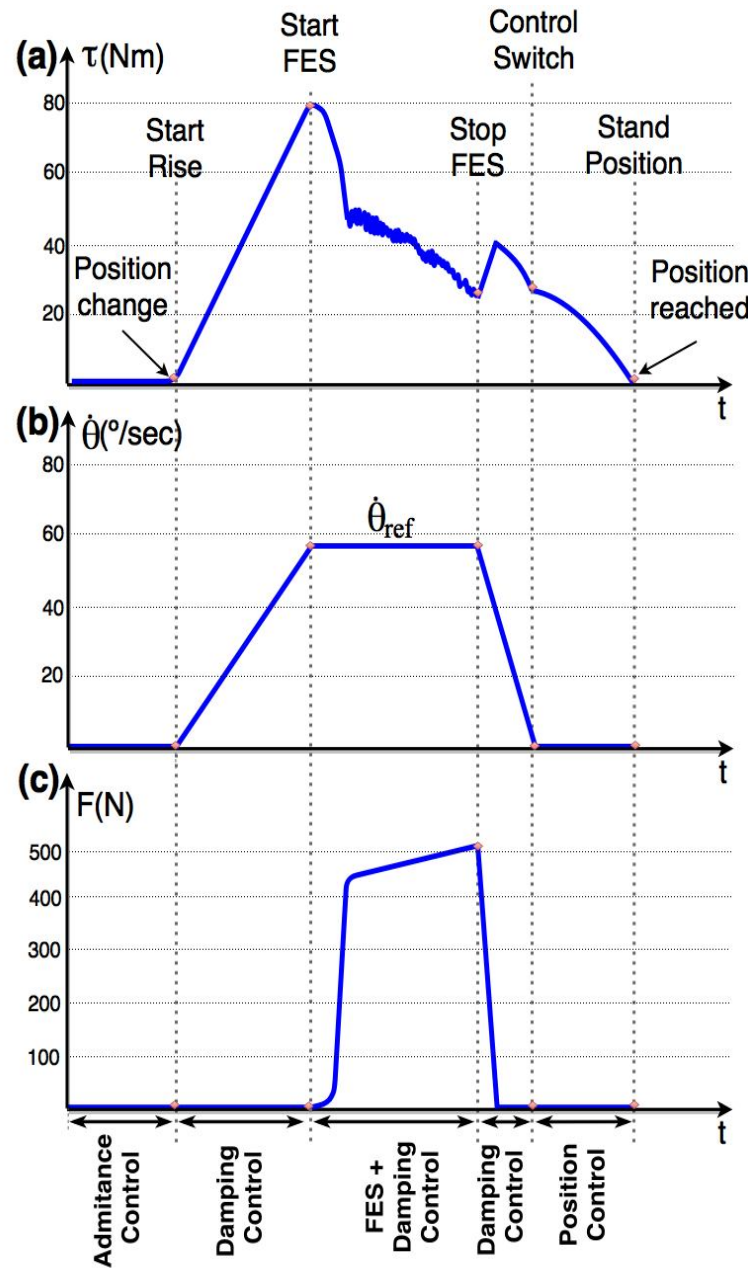

Figure 2. Knee joint behavior in the sit-to-stand task (a) Representation of motor torque behavior along time, (b) Reference velocity evolution, (c) Force generated by FES. 
joint to slow down before entering in the last stage. Here the deceleration can be higher than in take-off period, thanks to gravity direction.

Stage 3: Upright Posture - At this stage, the user must be assisted to the resting or final position while maintaining the postural stability. From arrival joint position at low speed, a position control with variable stiffness is applied:

$$
\tau=K_{s}\left(\theta_{\text {rest }}-\theta_{\text {act }}\right)
$$

where, $\tau$ is the applied torque to guide the joint, $K_{S}$ is the stiffness constant for the torque acting on the joint. $\theta_{\text {rest }}$ and $\theta_{\text {act }}$ are the rest goal position and current joint angle respectively. In this case, the stiffness $K_{S}$ is initially low to ensure good transition from the previous control mode, but it is increased along time quickly to its maximum value to maintain the stability at the end for keeping the user in upright position.

Fig.3 illustrates the control scheme for the implemented stiffness-damping approach. The series of the events in the sit-to-stand task combined with the changes in the joint velocity and position are considered in real-time.

\section{Simulation Analysis}

As a preliminary study, a simulation model was developed using the Matlab/Simulink SimMechanics toolbox. The simulation model has three revolute joints, emulating the behaviour of the hip, knee and ankle joint

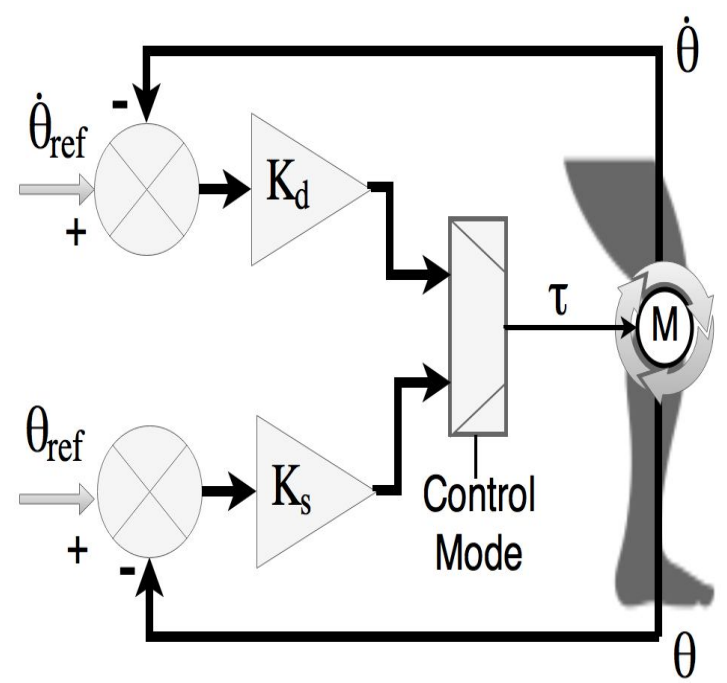

Figure 3. Schematic representation of the control model; The stiffness-damping control is considered in real-time to ensure the smooth transition.

${ }^{1}$ The exoskeleton used in this study was developed as part of the HYPER project (Hybrid Neuroprosthetic and Neurorobotic devices for functional compensation and rehabilitation of motor disorders), grant CSD2009-00067 CONSOLIDER INGENIO 2010 from MINECO (Spanish Ministry for Science and Education))

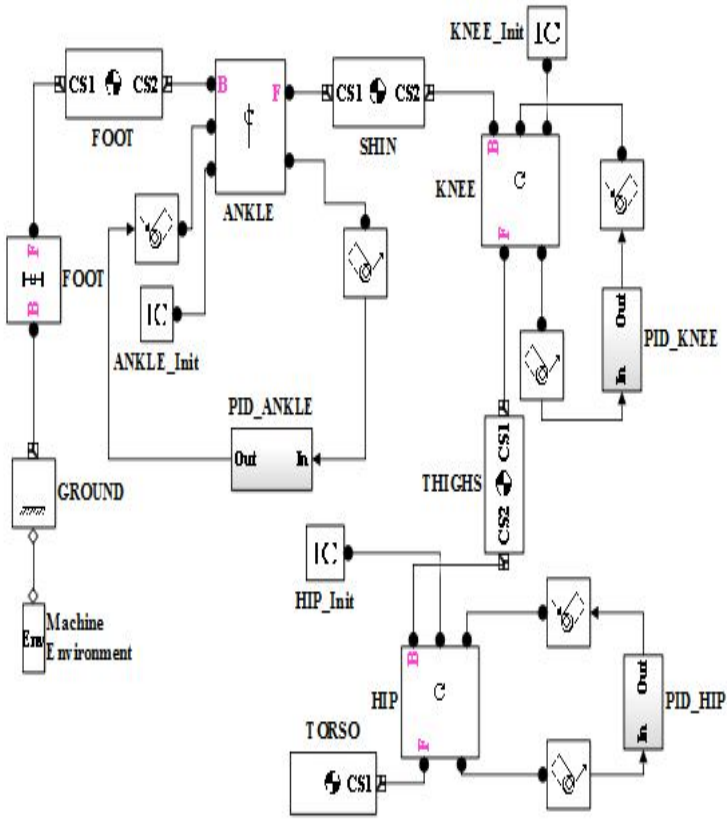

Figure 4. Simulation model of the Sit-to-stand transition

in the sit-to-stand transition. Each joint of the exoskeleton has 1 DoF (degree of freedom) revolute joint which can be driven by position and torque control respectively based on the therapy requirements. The mechanical parameters of the exoskeleton can be adjusted depending on the anthropomorphic parameters of each individual. Fig.4 shows the Simulink model of the exoskeleton developed based on the real mechanical considerations of the exoskeleton. The joints of the model are driven by PID control, based on the force applied which in turn is based on the error in its position/velocity. This Simulink model has been used in a previous study to analyze the effect of disturbances and the suitable method for recovering movement efficiently [12]. The model consists of 3 joints each driven by a 1 DoF revolute actuator which in turn is actuated by an implicit force control mechanism.

\section{EXOSKELETON H1}

$\mathrm{H} 1$ is a 6 DoF wearable lower limb orthosis with an anthropomorphic configuration to assist individuals with incomplete Spinal cord injury (SCI) or Stroke. The exoskeleton used in this study was built within the framework of the Hyper ${ }^{1}$ project. $\mathrm{H} 1$ has three joints for each leg: hip, knee and ankle, each joint is powered by a DC motor coupled with a harmonic drive gear. The exoskeleton is equipped with potentiometers and strain gauges to measure the joint angles and human-orthosis interaction torques on the links respectively. A detailed description about the exoskeleton structure and communication parameters is detailed in [14]. The variable stiffness control ensures a safe therapeutic experience $[1,12]$. The exoskeleton permits a stiffness value within the range of $1-100 \mathrm{Nm} / \mathrm{deg}$. A low 
stiffness value $(<10 \mathrm{Nm} / \mathrm{deg})$ will not cause any significant effect on the user's performance. Similarly, a high stiffness value $(>80 \mathrm{Nm} / \mathrm{deg})$ will provide a completely assisted movement, with few or no input from the user.

\section{Experimentation}

The experimentation was initially performed with a simulation experiment to study the controller performance and the joint torque behaviour. For the real study, the control was tested with healthy subjects wearing the exoskeleton such as to test the efficiency and the assisting capability of the proposed model. The initial stiffness value must be defined taking into account the user's capability and the degree of assistance to be applied by the orthosis. The initial reference velocity is assumed to be $0.5 \mathrm{~m} / \mathrm{s}$ which eventually provides the sufficient damping to make sure the model reaches to the target position with ample of time. The stiffness constant for the knee joint is considered as $50 \mathrm{Nm} / \mathrm{deg}$ for the healthy subjects. This stiffness provides sufficient assistance for the healthy subjects and suitable to let the users progress in their movement. In case of the paraplegic patients, this stiffness value must be maintained at a higher level in order to provide more assistance.

The transition can be differentiated or classified as the 4 step process.

1. The subject makes the effort to raise himself from the sitting position

2. The joints knee and ankle transfers from the initial position

3. The subject adjusts the hip joint rotation

4. The whole body is stabilised

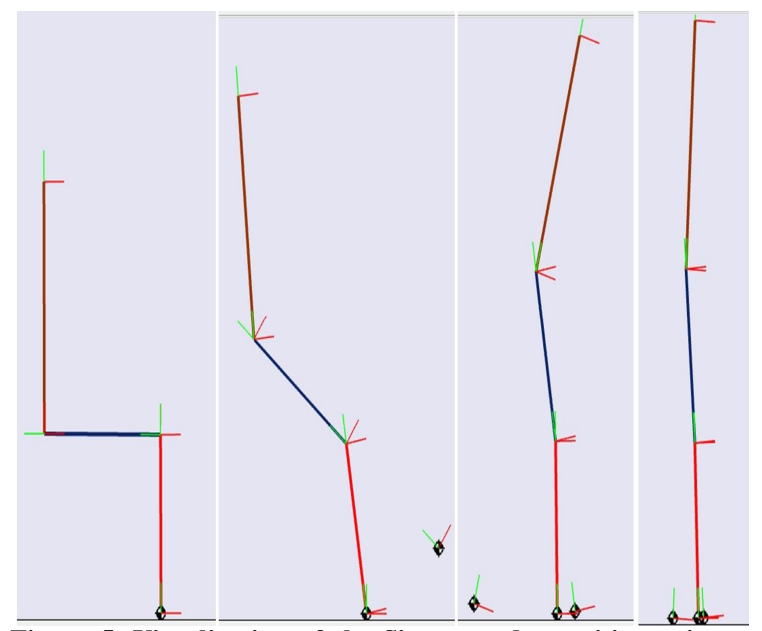

Figure 5. Visualization of the Sit-to-stand transition using a simulation model; The displacement of the COM in the different stages of the sit-to-stand transition can also be observed.
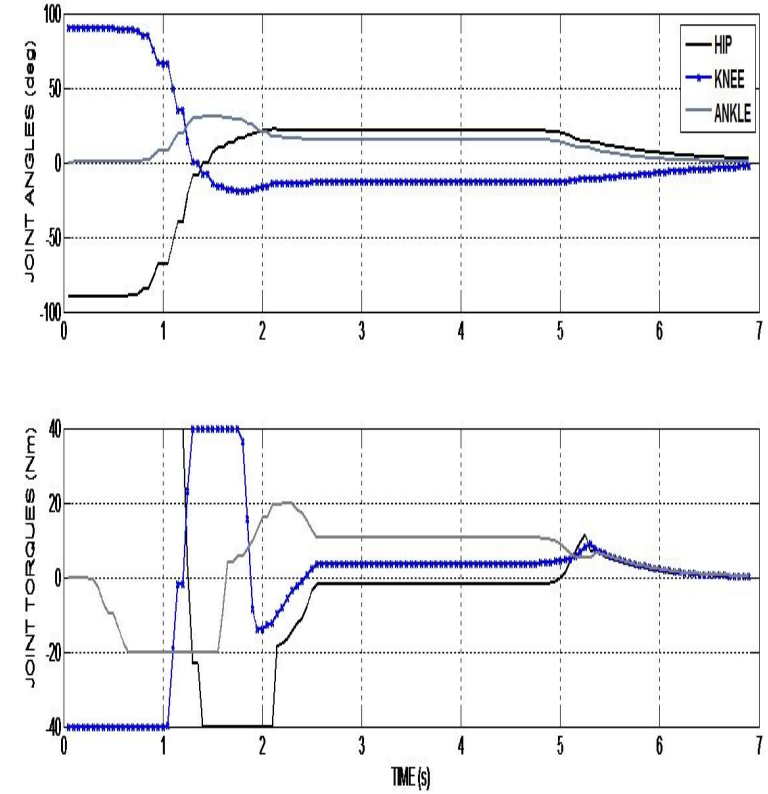

Figure 6. Simulation results of a sit-to-stand transition. The joint torques and angles demonstrates the joint performance in this transition.

\section{RESUlT AND DisCUSSION}

Sit-to-stand is a first step of transition in a lower limb rehabilitation task, to help the user to move from their wheel chair and to progress towards the next step in rehabilitation. This task has been evaluated using a stiffness-damping control. In order to provide the adequate support to the user, this task has been approached with a combination of three different control strategies. This test has been performed as a preliminary study with healthy subjects.

Fig.5, presents the visualization results of the different stages in sit-to-stand transition of the simulated model. Naturally, the hip movement should be in the beginning of the process followed by the flexion in the knee and ankle joints. The simulation model was relying in maintaining the hip position until it reaches the alternative ending of the flexed knee.

The simulation results presented in Fig.6, illustrates the joint torque needed in both the hip and knee joint in reaching the final stage of standing position. The initial joint angles of the hip and knee were assumed to be at 90deg and -90deg respectively which is a complete sitting position. However, in practice these initial stages can be depending on the user comfort and ability.

As shown in Fig.7, the transition of sit-to-stance can be classified as four events $\boldsymbol{A}$ to $\boldsymbol{D}$. The joint movement is initiated when the change in the joint movement is observed $(\boldsymbol{A})$. The assistance begins from $\boldsymbol{B}$ to the final $\boldsymbol{D}$, first with velocity-based control and a constant damping. The constant damping value supports the user evolution as needed, and ensures stability and assistance through the transition to standing. When the 


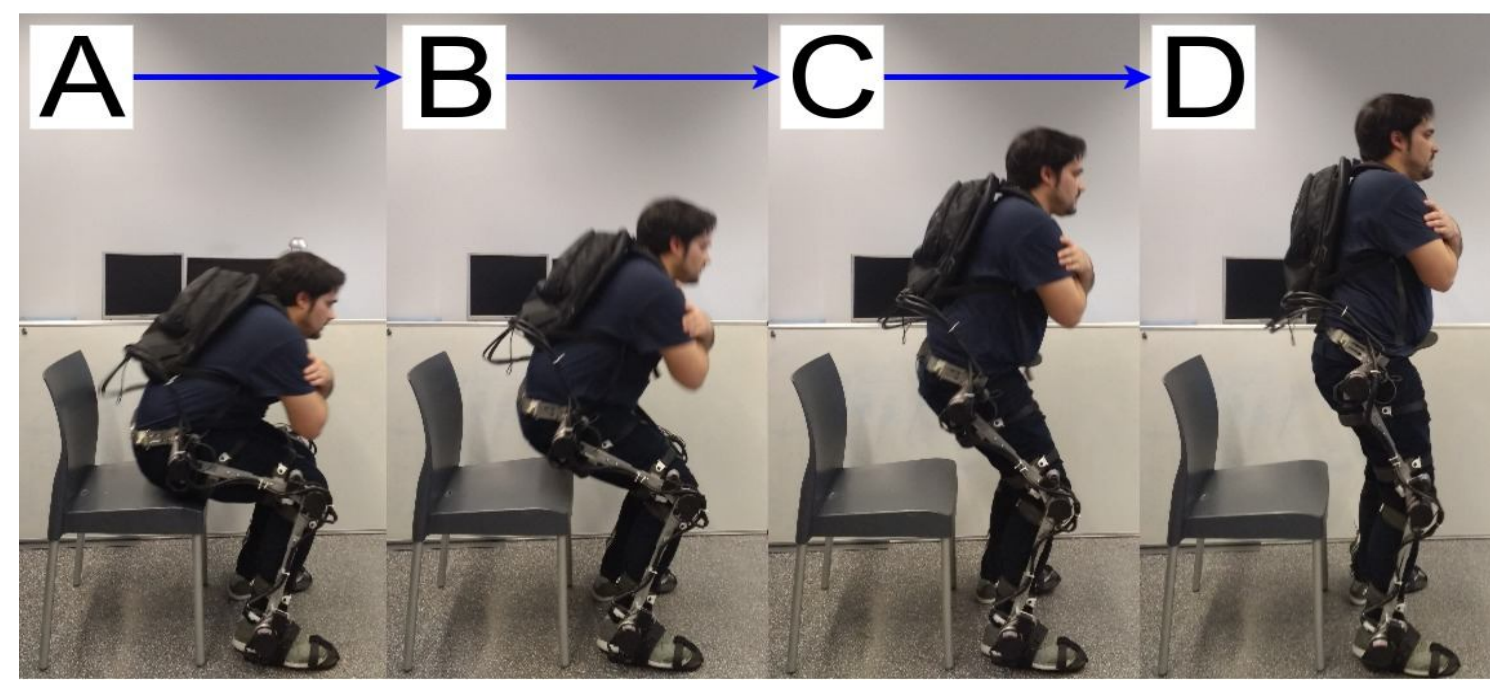

Figure 7. Sit-to-stand task performed by a healthy subject with the exoskeleton H1; Sit-to-stand task performed by a healthy subject with the exoskeleton $\mathrm{H} 1$

user reaches the standing position $(\boldsymbol{D})$ the control mode shifts to position control which helps in maintaining the equilibrium, with or without any external support. This kind of assistance is possible with a fixed stiffness to prevent the user from falling.

Fig.8 illustrates results on a healthy subject. The variation in the joint angles and interaction torques in the hip, knee and ankle joints are shown. A significant change in the knee joint movement is observed but supported by a large amount of interaction in the hip and ankle joint, with constant position. The movement occurs within a span of 1-3 seconds which is related to the velocity of the movement performed by the user.
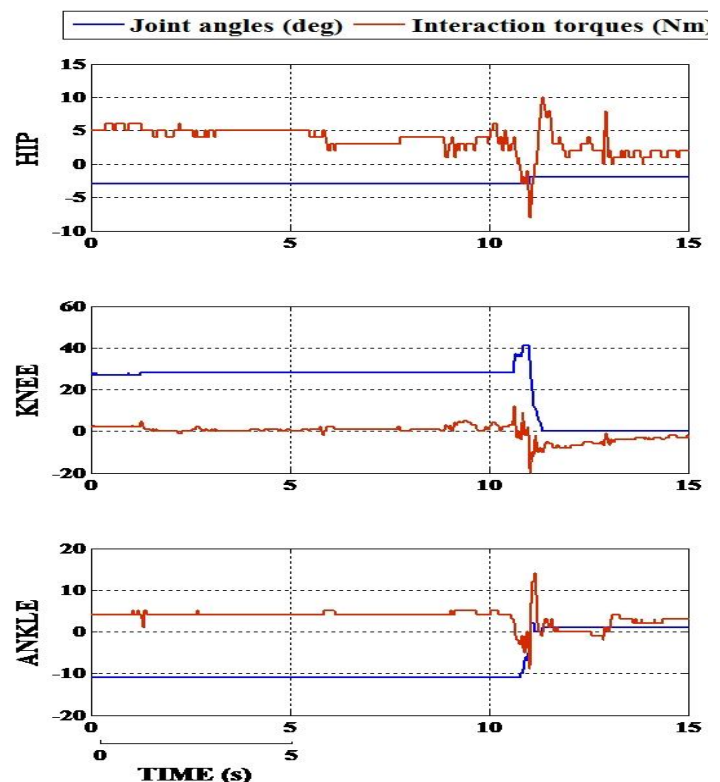

Figure 8. Changes in the joint angles and interaction torques after sit-to-stand transition performed by a healthy subject
For paraplegic patients, muscle stimulation might be needed to provide both muscle and functional assistance. The following are the sequential movements involved in case of experiment with patients, with the combination of FES:

1. From stage A to B with admittance control, for all the joints.

2. At B, FES activation must be initiated while the exoskeleton will maintain with a fixed damping behavior. This will help the muscle to move the user while the exoskeleton provides sufficient postural assistance.

3. At $\mathrm{C}$, the FES must be deactivated since the joint position is reached the standing phase. The joint damping must be maintained constant while decelerating to help the user maintain the posture.

4. At $\mathrm{D}$ is the end of the sequence, indicating the switch to position control model.

\section{CONCLUSION}

An event-based control model with a combination of stiffness-damping control adaptation has been presented for a sit-to-stand task in rehabilitation. The presented control model has been initially tested in a simulation environment and further progressed into a real study with a healthy subject using a wearable exoskeleton. The results of the simulation study and the real study has been presented discussing the efficacy of the system in handling the human interaction and the type of assistance provided in a rehabilitation task. The control model also provides the possibility of combining a muscle stimulation models for paraplegic individuals 
which is considered as the next objective of the presented work.

\section{References}

[1] V. Rajasekaran, J. Aranda and A. Casals, "An Adaptive control strategy for postural stability using a wearable exoskeleton," Robotics and Autonomous Systems, vol. 73, pp. 16-23, 2015.

[2] W. Huo, S. Mohammed, Y. Amirat and K. Kong, "Active Impedance Control of a Lower Limb Exoskeleton to Assist Sitto-Stand Movement," in IEEE International Conference on Robotics and Automation (ICRA), Stockholm, 2016.

[3] D. Chugo, H. Kaetsu, N. Miyake, K. Kawabata, H. Asama and K. Kosuge, "Force Assistance System for Standing-Up Motion," in Proceedings of the 2006 IEEE International Conference on Mechatronics and Automation, 2006.

[4] Y. Hirata, J. Higuchi, T. Hatsukari and K. Kosuge, "Sit-to-stand assist system by using handrail and electric bed moving up and down," in 2nd IEEE RAS/EMBS International Conference on Biomedical Robotics and Biomechatronics, 2008.

[5] S. R. Chang, M. J. Nandor, R. Kobetic, K. M. Foglyano, R. D. Quinn and R. J. Triolo, "Improving stand-to-sit maneuver for individuals with spinal cord injury," Journal of NeuroEngineering and Rehabilitation, vol. 13, no. 27, 2016.

[6] S. M. Taslim Reza, N. Ahmad, I. A. Choudhury and R. A. Raja Ghazilla, "A Fuzzy controller for lower limb exoskeletons during sit-to-stand and stand-to-sit movement using wearable sensors," Sensors, vol. 14, pp. 4342-4363, 2014.

[7] K. Kim, W. Cho, G. Yuk, H. Yang, B.-R. Jo and B.-H. Min, "Kinematic analysis of sit-to-stand assistive device for the elderly and disabled," in 2011 IEEE International Conference on Rehabilitation Robotics (ICORR), 2011.

[8] P. Médèric, V. Pasqui, F. Plumet and P. Bidaud, "Sit to Stand
Transfer Assisting by an Intelligent Walking-Aid," in Climbing and Walking Robots, Springer Berlin Heidelberg, 2005, pp. 1127-1135.

[9] K. Junius, B. Brackx, V. Grosu, H. Cuypers, J. Geeroms, M. Moltedo, B. Vanderborght and D. Lefeber, "Mechatronic Design of a sit-to-stance Exoskeleton," in IEEE RAS/EMBS 5th International Conference on Biomedical Robotics \& Biomechatronics (BioRob), São Paulo, Brazil, 2014.

[10] R. J. Wong and J. A. Smith, "Regenerative effects in the Sit-toStand and Stand-to-Sit movement," Robotica, vol. 33, pp. 107$126,2015$.

[11] A. Kralj, R. Acimovic and U. Stanic, "Enhancement of hemiplegic patient rehabilitation by means of functional electrical stimulation," Prosthetics and Orthotics International, vol. 17, no. 2, pp. 107-114, 1993.

[12] V. Rajasekaran, J. Aranda and A. Casals, "Recovering planned trajectories in robotics rehabilitation therapies under the effect of disturbances," International Journal on System Dynamics and Applications (IJSDA), vol. 3, no. 2, pp. 34-49, 2014.

[13] A. J. Del-Ama, A. Gil-Agudo, E. Bravo-Esteban, S. PerezNombela, J. L. Pons and J. C. Moreno, "Hybrid therapy of walking with Kinesis overground robot for persons with incomplete Spinal Cord Injury: A feasibility study," Robotics and Autonomous Systems, vol. 73, pp. 44-58, 2015.

[14] M. Bortole, A. J. Del-Ama, E. Rocon, J. C. Moreno, F. Brunetti and J. L. Pons, "A Robotic Exoskeleton for Overground Gait Rehabilitation," in IEEE International Conference on Robotics and Automation, 2013. 